\title{
Comparison of Different Model Updating Algorithms to Detect Damage in A Structural Slab Using Mode Shape Data
}

\author{
Lyn Dee Goh", ${ }^{1, *}$ Norhisham Bakhary², Fatin Nadiah Abdul Rahman² \\ ${ }^{1}$ School of Civil Engineering, College of Engineering, Universiti Teknologi MARA, Cawangan Pulau Pinang, 13500 Permatang Pauh, \\ Pulau Pinang, Malaysia \\ ${ }^{2}$ School of Civil Engineering, Faculty of Engineering, Universiti Teknologi Malaysia, 81310 Johor, Malaysia
}

Received June 21, 2021; Revised October 19, 2021; Accepted November 17, 2021

\section{Cite This Paper in the following Citation Styles}

(a): [1] Lyn Dee Goh, Norhisham Bakhary, Fatin Nadiah Abdul Rahman , "Comparison of Different Model Updating Algorithms to Detect Damage in A Structural Slab Using Mode Shape Data," Civil Engineering and Architecture, Vol. 10, No. 1, pp. 376-387, 2022. DOI: 10.13189/cea.2022.100132.

(b): Lyn Dee Goh, Norhisham Bakhary, Fatin Nadiah Abdul Rahman (2022). Comparison of Different Model Updating Algorithms to Detect Damage in A Structural Slab Using Mode Shape Data. Civil Engineering and Architecture, 10(1), 376-387. DOI: 10.13189/cea.2022.100132.

Copyright@2022 by authors, all rights reserved. Authors agree that this article remains permanently open access under the terms of the Creative Commons Attribution License 4.0 International License

Abstract Vibration-based damage detection (VBDD) is one of the techniques used in Structural Health Monitoring (SHM) for detecting structural damage. VBDD provides more efficient, reliable and economical methods to improve the safety and reduce the cost of maintenance in structural engineering. This paper investigates the performance of model updating (MU) method using different types of algorithms in damage detection. A finite element analysis is performed to obtain dynamic properties of undamaged and damaged slab structure for model updating process using different types of algorithms. Three optimisation functions of different algorithms employed in this study are constrained optimisation, least-square optimisation and multiobjective optimisation. Different damage cases are introduced at different locations with different damaged intensities by reducing the structure's elastic modulus in corresponding segment. The performance of MU is evaluated by Stiffness Reduction Factor (SRF). The comparison of SRF for actual value and predicting value is made to check the applicability of three types of algorithms in damage detection. Mean Square Error (MSE) is applied to evaluate stopping and tolerance criteria effect on result improvement. The results show all three types of algorithms are capable to provide reliable results in damage prediction and an improvement of result has been made when increasing the stopping and tolerance criteria.

Keywords Damage Detection, Model Updating, Optimisation Algorithms

\section{Introduction}

Recently, researchers have developed various types of techniques to produce a reliable approach to improve the safety and reduce the cost of maintenance in structural engineering. According to [1], SHM is a procedure of employing a damage detection strategy. In the procedure, the capability of the structure information is fed to enquire its desired function in the future due to degradation and caused by environmental impact. It applies the concept of degradation of structural design and study on the changes in dynamic properties for instance natural frequencies, mode shapes and damping where the degradation of the structure is represented by the changes in material and geometric properties. Damage is defined as the material change and/or structural geometric properties change including the changes to the boundary condition and system which influenced the performance of the system [2]. The damage cannot be express if there are no 
significant differentiations can be made between two different states of the system. Numerous studies have been conducted to verify the abilities of dynamic properties in detecting and locating the damage [3-4].

Based on previous research, traditional methods that have been used to quantify the damage are either visual or local experimental methods, for example magnetic field methods, thermal field methods, radiograph, eddy-current methods and ultrasonic methods [5]. However, these methods are limited to local damages and only applicable at accessible area or near surface area of the structure. Due to the limitation, the VBDD method which applies the global damage identification technique is developed to overcome these shortcomings and difficulties.

Numerous and continuous studies have been conducted to improve the performance of VBDD in damage detection. A large number of techniques, methods and algorithms have been developed to overcome the problems arisen in analysing the complex and also basic structural system. Several methods used in VBDD methods are:

- Damage detection based on changes in basic modal properties

- Mode shape curvature changes

- Model updating based method

Damage detection based on changes in basic modal properties essentially uses basic modal properties comprising of resonant frequencies, modal damping and mode shape vector. By using frequency change, the application for damage identification has significant practical limitations due to low sensitivity to damage as it needs a large level of damage in order to get precise measurement [6], beside requiring a large number of measurements. Studies have showed that resonant frequency has much less statistical variation from random error compared to other modal parameters. However, changes of modal frequencies in damage detection provide insufficient data to determine the location of the damage uniquely.

The concept of mode shape curvature changes method is defined by the change of curvature from derivation of mode shape. This derivation is obtained using second derivative of mode shape vector that is more sensitive to small perturbation in system [7]. Hence, there is a direct relationship between curvature and bending strain for beam, plate and shell. Changes in curvature can provide damage identification result for FEM beam structure and the curvature is computed from the displacement mode shape using the central difference operator. However, reviewed by [6] discovered that calculating curvature from mode shape numerically resulted in unacceptable error and the improvement of the result can be obtained using measured strain instead of curvature.

Another class in VBDD is model updating-based method. This method is based on the concept of comparison between modal parameter obtained from numerical model of FE modelling and experimental model from vibration tests. The model updating is a process of adjusting certain parameters of finite element model until it closely matched with the measured modal data. Study by [8] showed that speed of iteration in damaged detection is successfully achieved by the applicability of nonlinear least squares minimisation with the gauss-Newton algorithms using the frequency domain.

Modal updating method is the focus of this study. The model updating is performed iteratively to minimise the discrepancies between two finite element models for damage detection. Model updating using sensitivity method is utilised in this study because it is capable to provide more reliable results according to previous sensitivity studies conducted. Finite element model (FEM) is utilised to generate the modal data required in model updating process. According to [9], model updating is basically a process of adjusting selected variables of the FEM until an objective function is met. The selection of updating variables and the construction of the objective function are the critical part in model updating in order to provide better convergence. This process of model updating is repetitive until the differences between the objective function become smaller than consecutive iteration. Generally, the damage detection is nonlinear function of selected updating parameters and the inverse process that needs the application of algorithms for evaluation.

This study aims to investigate the applicability of modal updating method for damage detection of a single span slab structure. In addition, this study also investigates the efficiency and effects of different types of modals updating algorithms as well as the effect of different tolerances values for damage detection based on modal data. Thus, this study demonstrates the applicability of the vibration data, precisely, modal domain data, for damage detection purpose. In conventional model updating-based method, the FEM is required for sensitive computation during the iteration process which leads to high time consumption and slow convergence. Due to the less practical of conventional methods, in conjunction with SDTools, FEM updating has been alternatively built in simpler manner and timeefficiently processed where conventional FE model is replaced with SDT model.

In this study, single objective function using mode shape, is utilised. In comparison with frequency, mode shape has higher sensitivity in producing better damage localisation. In addition, selection of algorithms also contributes to a large role in providing reliable damage localisation. Thus, this study employs three different algorithms using model updating in damage detection. The three different algorithms are constrained optimization (fmincon), least-square optimization (Isqnonlin) and multiobjective optimization (fgoalattain) functions. However, due to the limitation in optimisation 
termination criteria provided at default condition, this study provides the extension to the damage detection purpose in which, variation of tolerances is applied in damage cases. The tolerances consist of $(1 e-6)$ (default), $(1 e-10),(1 e-15)$ and $(1 e-20)$. Hence, this study proposed a new model updating method employing SDT for damage detection by considering both variation of algorithms and tolerances for better damage detection purpose.

\section{Theoretical Background}

VBDD is a non-destructive evaluation of structural analysis which offers more efficient and reliable methods to detect damage at the earliest stage. VBDD overcomes the drawback of experimental method whereby the damage detection is generally able to evaluate near to the structural surface area and require the damage locality known as a priori. Nowadays, VBDD has been widely used in many engineering sectors such as civil, mechanical and aerospace to examine changes in structure on the modal parameters. According to [1], earlier studies of VBDD are in oil industry to examine the damage in offshore platform. Reference [10] describes many factors that contribute to the increasing of vibration-based damage identification method application. The main factor is the failures of structures and mechanical systems used by the public.

The basic concept of VBDD is any damage occurs in physical properties of the structure will promote the changes of modal properties. The physical properties that can influence the modal properties consisting of natural frequencies, mode shapes and modal damping are mass, stiffness and damping. The equation of motion in (1) shows the relationship of physical properties modal properties that applies in VBDD:

$$
\mathrm{M} \ddot{x}+\mathrm{C} \dot{x}+\mathrm{K} x=0
$$

where $\mathrm{M}$ is the mass matrix, $\mathrm{C}$ is the damping matrix and $\mathrm{K}$ is the stiffness matrix. $\ddot{x}, \dot{x}$ and $x$ are vectors representing acceleration, velocity and displacement, respectively. The associated eigenvalue problem is shown in (2):

$$
\left(\omega_{i}^{2} \mathrm{M}+j \omega_{i}^{2} \mathrm{C}+\mathrm{K}\right) \phi_{i}=0
$$

where $\omega_{i}^{2}$ and $\phi_{i}$ are the $i^{\text {th }}$ modal circular frequency and mode shape, respectively, and $j$ is the imaginary unit.

There are three types of data utilised in VBDD which can be classified as time, frequency and modal domains. In time domain, the respond can be measured as time history using displacement, velocity and acceleration from the vibration test using sensors positioned in the structure. The ARMA model and ARMAX model are vibrating systems used to analysis time series. Reference [6] stated that the problem of structural dynamics is difficult to analyse using time domain due to a high number of degrees of freedom. Hence it requires an appropriate reduction method of data. The numerous volumes of data from time domain can be reduced and converted by using Fast Fourier Transform algorithm to Frequency Respond Data (FRF) which can be classified as frequency domain. In review by [6] stated that in further analysis, the extraction of frequency data domain is carried out to produce modal quantities namely natural frequencies, mode shape and modal damping values as modal domain data. Besides, many literatures have concentrated the method of using modal domain due to: (i) the early literature concentrates on modal domain, and (ii) natural frequencies and mode shape can provide more attractive than abstract characteristics from frequency and time domains. The analysis of modal domain is also easier to interpret due to a smaller amount of data produced.

Model updating can be classified into 2 categories which are direct method (non-iterative) and sensitivity method (iterative). Direct method is the computational of mass matrix and stiffness matrix using closed-form direct solution. The problem is generally formulated as a Lagrange multiplier or penalty-based optimisation [11]. Study by [12] concluded that the detailed correlation procedure is required to establish a set of reliable test data as postprocessor to verify the accuracy improvement and physical validation of the updated model.

Sensitivity (iterative) method is the most popular method and widely used in damage detection due to the capabilities in solving inverse problems. According to [13], the more preciseness and completeness in measured data and accuracy in numerical model can be achieved through (i) higher modes of numerical matrix system presented by stiffness matrix provide realistic stiffness model and (ii) the parameters of numerical model are related directly to geometry and material properties of structure may provide a meaningful model which direct method are not capable to.

The procedure of iterative method can be obtained in [14]. Selection of parameters to minimise the objective function has become the most crucial part in optimisation to ensure the effectiveness of model updating process. A sensitive design parameter is required in model updating to compute the correlation between two different types of system which are from analytical data and measured data in objective function.

The difference between various sensitivity-based FEM updating approaches in previous studies is (i) objective function, (ii) numerical approach used to implement the optimization and (iii) algorithms to obtain the sensitivity matrix [15]. Performance of model updating process greatly depends on the selection of residual in the objective function [16]. Study in [17] used MAC to evaluate the correlation between the experimental and analytical mode shape in objective function and the utilization of elastic modulus, $E$, and moment of inertia, $I_{y}$, as updating parameters provide a good result in damaged 
detection. While study by [18] proved that the objective function considering 11 measured frequencies with 10 updating structural parameters also can produce reliable result in damage detection of $1 / 150$ scaled suspension bridge model.

\section{Methodology}

There are 2 major parts in this study: i) Development of numerical model of a reinforced concrete slab using FEM and ii) model updating process using (fmincon), (Isqnonlin) and (fgoalattain) functions. There are two numerical models of the reinforced concrete slab generated, viz. for undamaged structure and damaged structure. Mode shapes data generated from the numerical models are utilized for the damage detection through the model updating process.

The numerical model is a two span simply supported reinforced concrete slab with a dimension $6400 \mathrm{~mm} \times 800$ $\mathrm{mm}$. The thickness of the lab is $100 \mathrm{~mm}$. The length of each span is $3000 \mathrm{~mm}$ and $200 \mathrm{~mm}$ overhang at both ends, after the supports. Material properties used are Young modulus, $E=3.3 \times 10^{10} \mathrm{~N} / \mathrm{mm}^{2}$, density, $\rho=2.45 \times 10^{3}$ $\mathrm{kg} / \mathrm{mm}^{3}$ and Poisson's ratio, $v=0.2$. The slab is considered as shell elements with 81 nodes and 52 elements and divided into seven symmetrical segments. Fig. 1 illustrates the reinforced concrete slab adopted in this study. Three damage cases are introduced at different locations with different damaged intensities by reducing the modulus of elasticity $(E)$ value for corresponding segment as shown in Table 1.

Due to difficulties in obtaining higher modes in real practice, only the first three mode shapes are considered in the model updating. The mode shapes are measured on the centreline along the span. Fig. 2 illustrates the mode shapes of undamaged slab for first three modes. Using the similar methodology, the steps are repeated for damage Case 1, Case 2 and Case 3 to obtain the value of the first natural frequencies. These natural frequencies are tabulated in Table 2. It is to note that Case 1 and Case 2 are single damaged cases with different damage intensities while Case 3 is multiple damaged cases. In comparison between Case 1 and Case 2, the average frequencies change for Case 2 is higher than Case 1 due to higher damage intensities introduced as shown in Table 1.

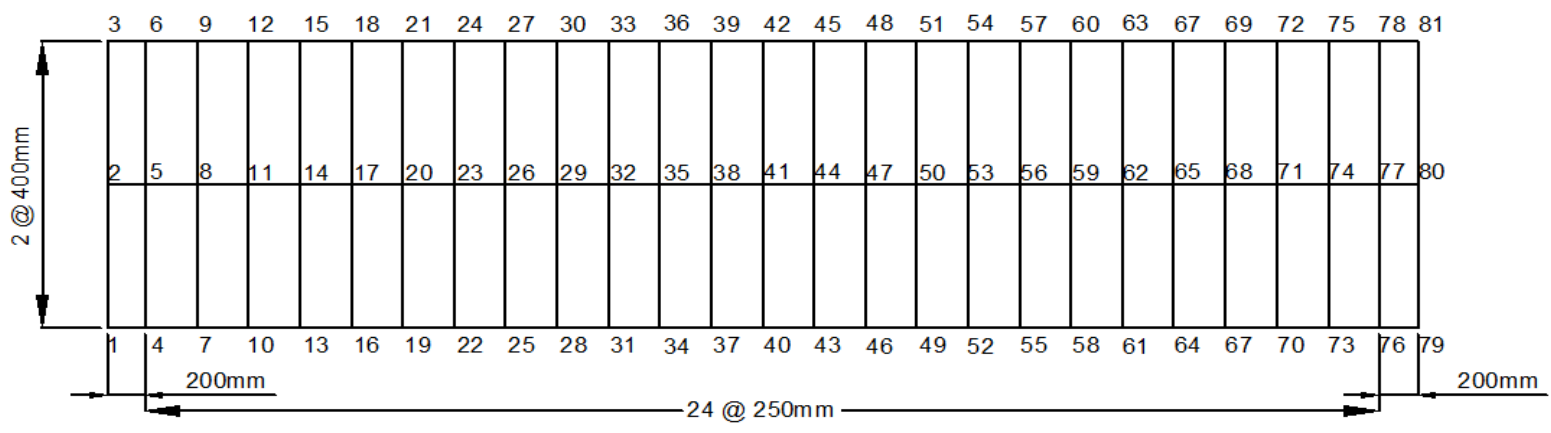

i) Finite element mesh with node number

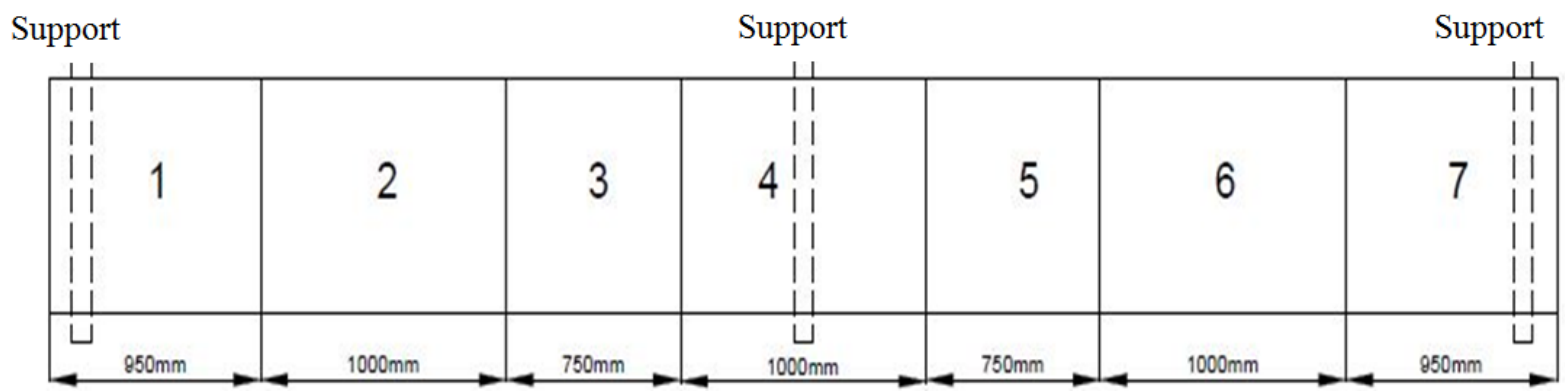

ii) Slab segment

Figure 1. Plan view of the reinforced concrete slab

Table 1. Stiffness $(E)$ values for Case 1, Case 2, and Case 3

\begin{tabular}{|c|c|c|c|c|c|c|c|}
\hline Segment & 1 & 2 & 3 & 4 & 5 & 7 \\
\hline Undamaged & $\mathrm{E}$ & $\mathrm{E}$ & $\mathrm{E}$ & $\mathrm{E}$ & $\mathrm{E}$ & $\mathrm{E}$ \\
\hline Case 1 & $\mathrm{E}$ & $0.7 \mathrm{E}$ & $\mathrm{E}$ & $\mathrm{E}$ & $\mathrm{E}$ & $\mathrm{E}$ \\
\hline Case 2 & $\mathrm{E}$ & $\mathrm{E}$ & $\mathrm{E}$ & $\mathrm{E}$ & $\mathrm{E}$ & $0.5 \mathrm{E}$ & $\mathrm{E}$ \\
\hline Case 3 & $\mathrm{E}$ & $\mathrm{E}$ & $0.7 \mathrm{E}$ & $\mathrm{E}$ & $\mathrm{E}$ & $0.7 \mathrm{E}$ \\
\hline
\end{tabular}


Table 2. First three natural frequencies of the slab in different cases (in $\mathrm{Hz}$ )

\begin{tabular}{|c|c|c|c|c|}
\hline & Undamaged & Case 1 & Case 2 & Case 3 \\
\hline $\mathbf{1}^{\text {st }} \mathbf{m o d e}$ & 18.636 & 17.571 & 16.399 & 17.057 \\
\hline $\mathbf{2}^{\text {nd }} \mathbf{m o d e}$ & 29.355 & 28.185 & 27.314 & 27.985 \\
\hline $\mathbf{3}^{\text {rd }} \mathbf{m o d e}$ & 76.213 & 74.233 & 71.868 & 71.813 \\
\hline
\end{tabular}

\section{Mode 1: $18.636 \mathrm{~Hz}$}

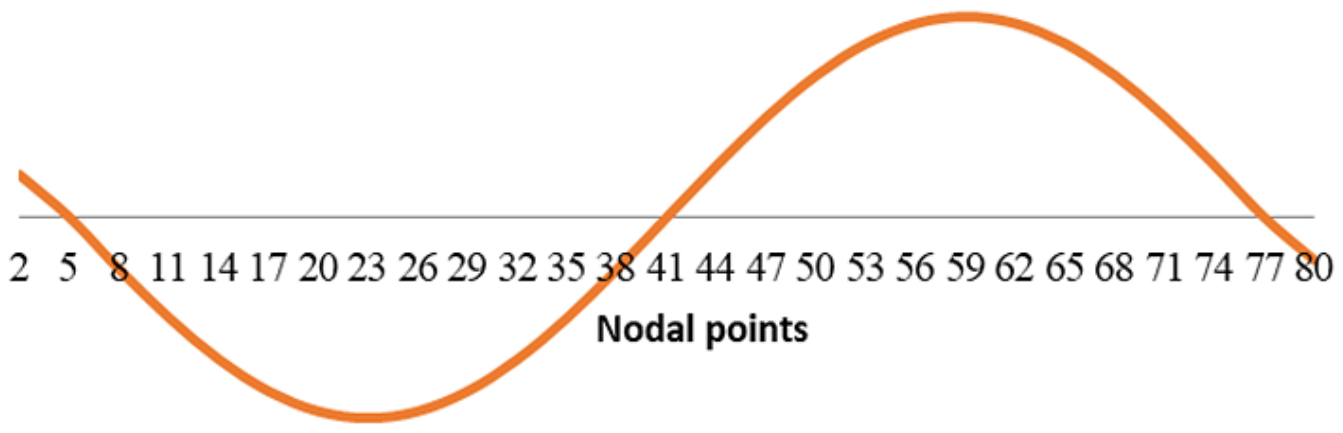

Mode 2: $29.355 \mathrm{~Hz}$

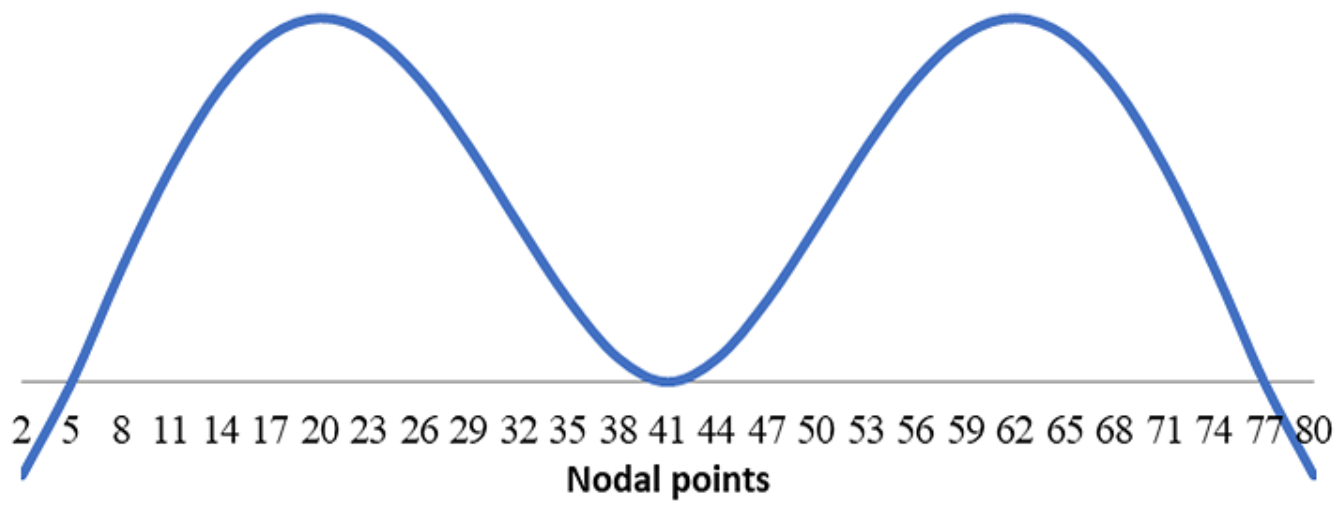

\section{Mode 3: $76.213 \mathrm{~Hz}$}

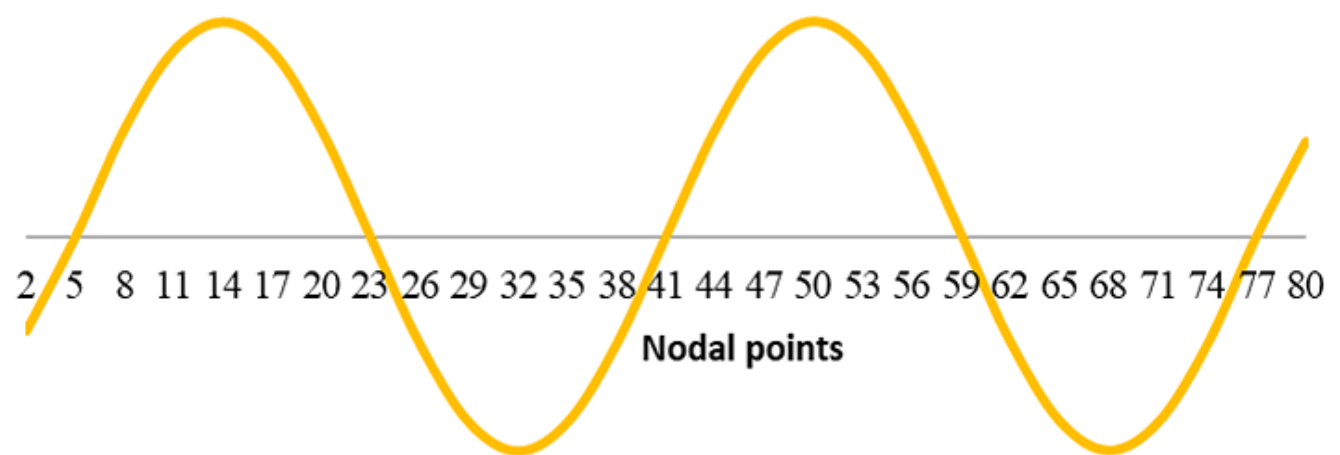

Figure 2. First three mode shapes of undamaged slab 
After completing generating the $E$ values from the numerical models, these $E$ values are used as the updating parameters together with the mode shapes generated. Subsequently, the Modal Assurance Criterion (MAC) evaluation is employed as objective function using different algorithms. The fmincon optimisation, Isqnonlin optimisation and fgoalattain optimisation functions are applied and they are presented in (3), (4) and (5), respectively, with (6) as the objective function. MAC provides a measure of consistency between mode shapes vectors from a finite element analysis. The predictions from model updating are executed using (7).

$$
\begin{gathered}
\min _{x}\left\{\begin{array}{c}
F(x) \leq 0 \\
\mathrm{l}_{\mathrm{b}} \leq \mathrm{x} \leq \mathrm{u}_{\mathrm{b}}
\end{array}\right. \\
\min _{x}\left\{\begin{array}{c}
\frac{1}{2}\|F(x)\|_{2}^{2}=\frac{1}{2} \sum_{i} f_{i}(x)^{2} \leq 0 \\
\mathrm{l}_{\mathrm{b}} \leq \mathrm{x} \leq \mathrm{u}_{\mathrm{b}}
\end{array}\right. \\
\min _{x}\left\{\begin{array}{c}
F(x)-w \cdot \gamma \leq \text { goal } \\
\mathrm{l}_{\mathrm{b}} \leq \mathrm{x} \leq \mathrm{u}_{\mathrm{b}}
\end{array}\right. \\
F(x)=1-M A C \\
\operatorname{MAC}(\mathrm{r}, \mathrm{q})=\frac{\left|\left\{\phi_{A}\right\}_{r}^{T}\left\{\phi_{X}\right\}_{q}\right|}{\left(\left\{\phi_{A}\right\}_{r}^{T}\left\{\phi_{A}\right\}_{r}\right)\left(\left\{\phi_{X}\right\}_{q}^{T}\left\{\phi_{X}\right\}_{q}\right)}
\end{gathered}
$$

$\left\{\phi_{A}\right\}$ is a mode shape vector of damaged from finite element analysis and $\left\{\phi_{x}\right\}$ is a mode shape vector of analytical predictions from modal updating process. From (5), $w$ is the weighting vector to control the attainment factor of the goals; $\gamma$ is a slack variable used as a dummy argument in the optimisation. The $l_{b}$ and $u_{b}$ in (3), (4) and (5) are the lower and upper bounds of design parameters.

The Modal Scale Factor (MSF) has been applied to mode shape vectors to normalise all estimate vectors that take into account the magnitude and the phase differences. The mode shapes vectors from a finite element analysis can be compared and contrasted with those from modal updating using (8).

$$
\operatorname{MSF}(c d r)=\left\{\Psi_{c r}\right\}\left\{\Psi_{d r}\right\} /\left\{\Psi_{d r}\right\}^{T}\left\{\Psi_{d r}\right\}
$$

$\left\{\Psi_{c r}\right\}$ is modal vector for reference c, mode $\mathrm{r}$ and $\left\{\Psi_{d r}\right\}$ is modal vector for reference $d$, mode $r$. The undamaged mode shapes vector is used as reference vector for MSF. Fig. 3 shows the mode shape 1 in undamaged and damaged cases before applying the MSF, while Fig 4 . presents the output after applying the MSF to all the estimate vectors.

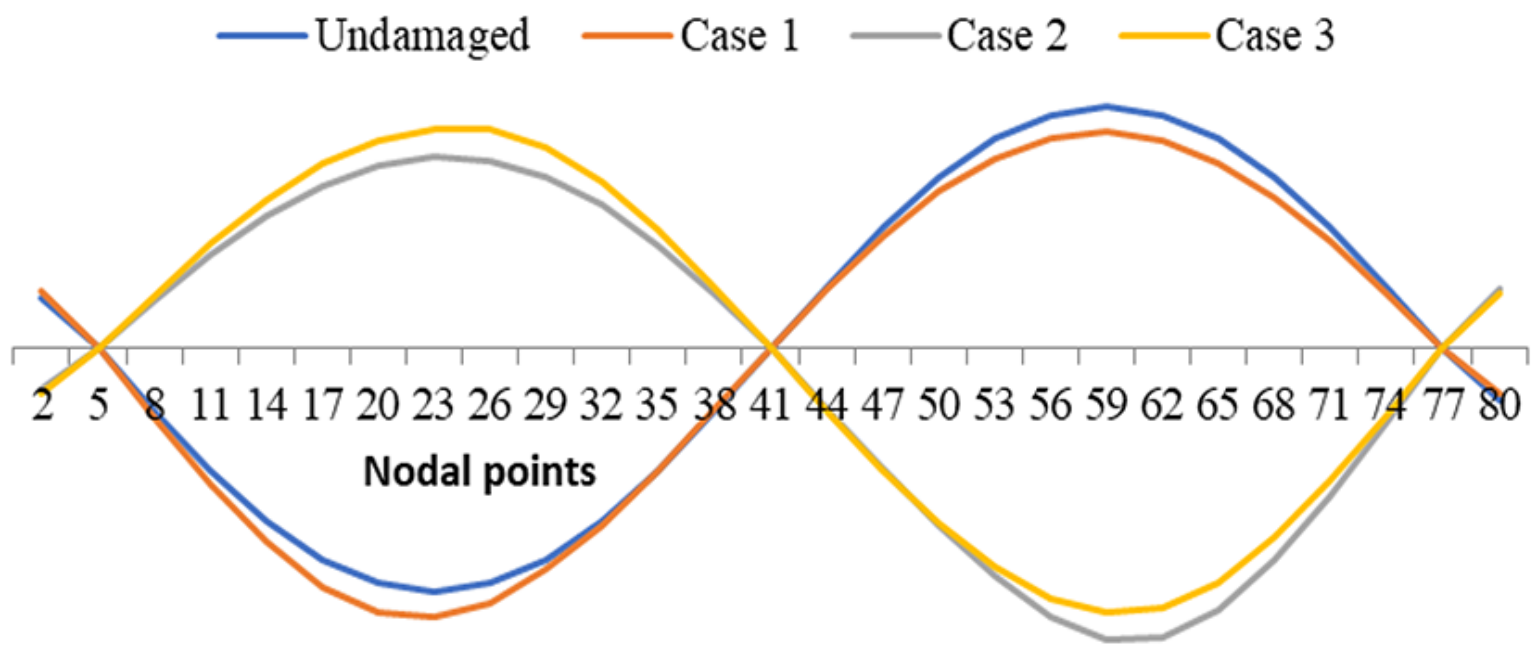

Figure 3. Mode shape 1 of different damage cases before normalisation 


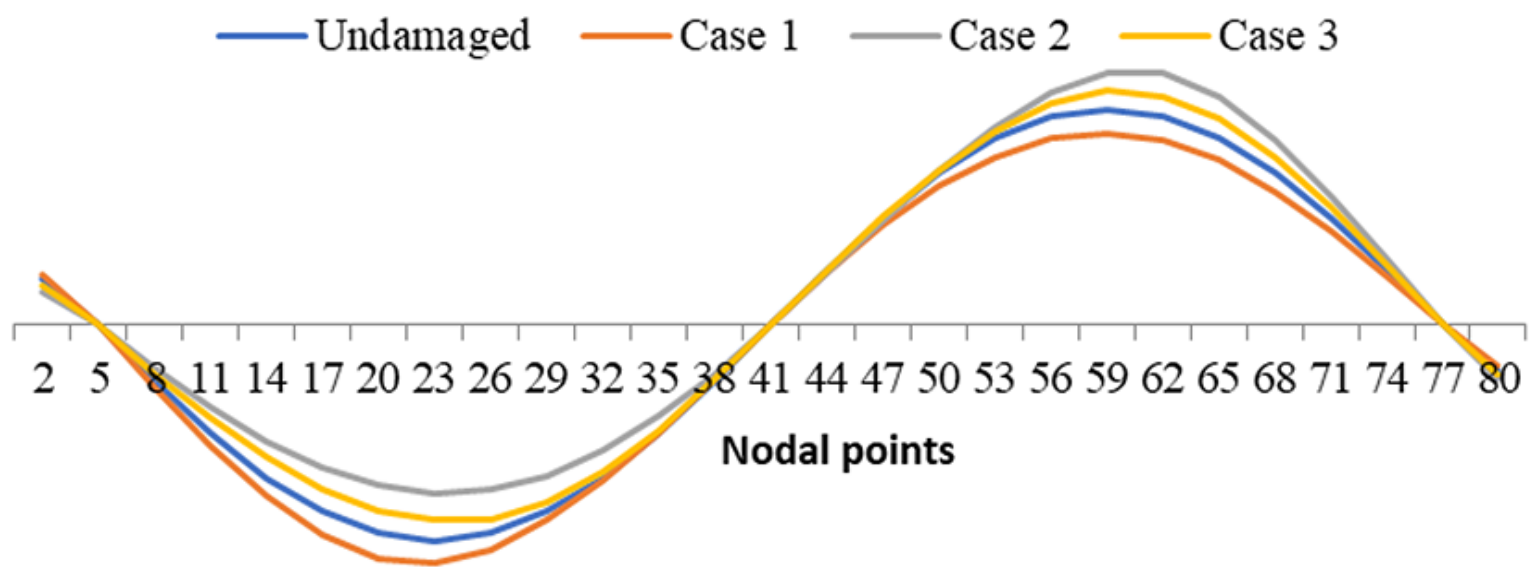

Figure 4. Mode shape 1 of different damage cases after normalisation

The result from model updating is the $E$ value in every segment. The changes in $E$ values are therefore used to detect and locate the damage of the slab structure using stiffness reduction factor (SRF) as presented in (9).

$$
S R F=\left(E_{\text {undamaged }}-E_{\text {damaged }}\right) /\left(E_{\text {undamaged }}\right)
$$

\section{Result and Discussion}

To examine the applicability of three different algorithms for damage detection, the model updating is performed using fmincon function, Isqnonlin function and fgoalattain function. A comparison between the models is conducted using the default tolerance and higher tolerance in different algorithms in all damaged cases for the model updating. Fig. 5, Fig. 7 and Fig 9 show the comparison between actual and predicted SRF values for Case 1, Case 2 and Case 3, respectively, when the default tolerances are applied in the procedure. Meanwhile Fig. 6, Fig. 8 and Fig. 10 present the improvement of performance after applying higher tolerance criteria in the similar cases.

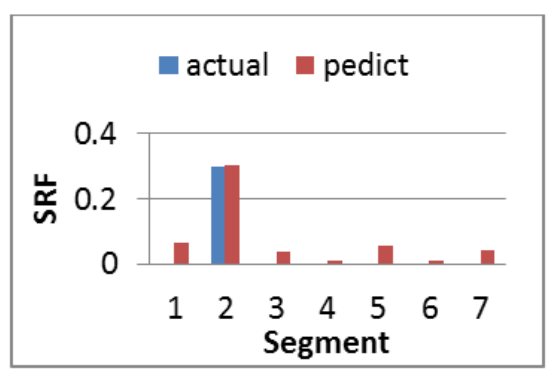

a) fmincon function

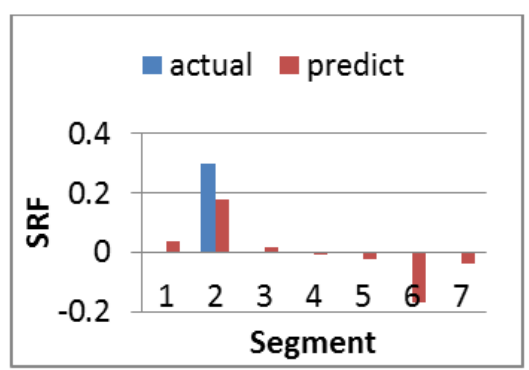

b) Isqnonlin function

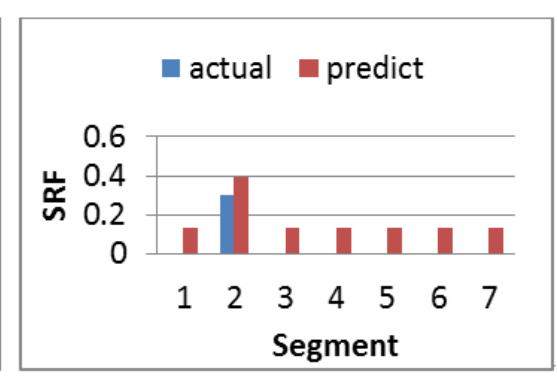

c) fgoalattain function

Figure 5. Performance of model updating for Case 1 using different function for default tolerance

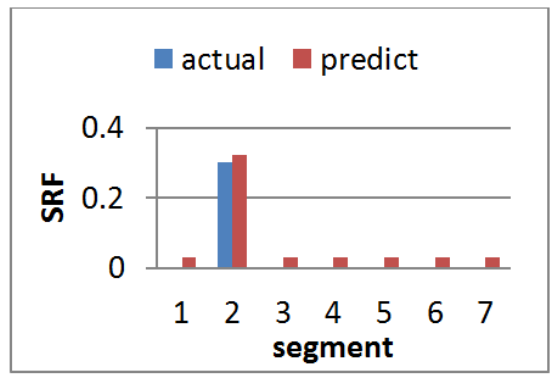

a) fmincon function

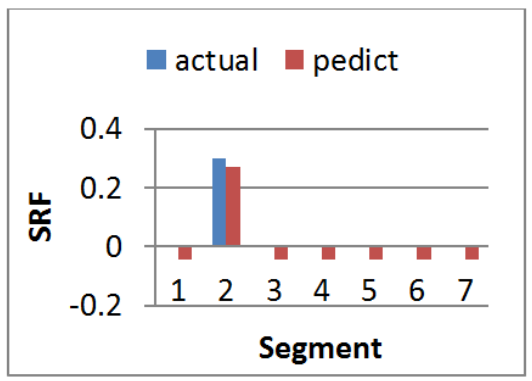

b) Isqnonlin function

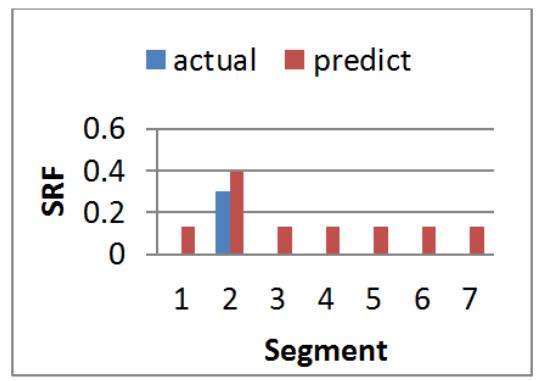

c) fgoalattain function

Figure 6. Performance of model updating for Case 1 using different function for higher tolerance 


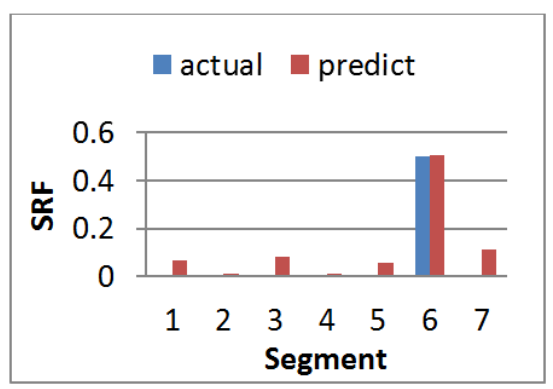

a) fmincon function

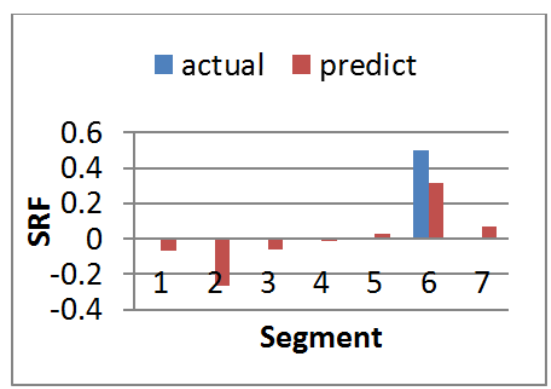

b) lsqnonlin function

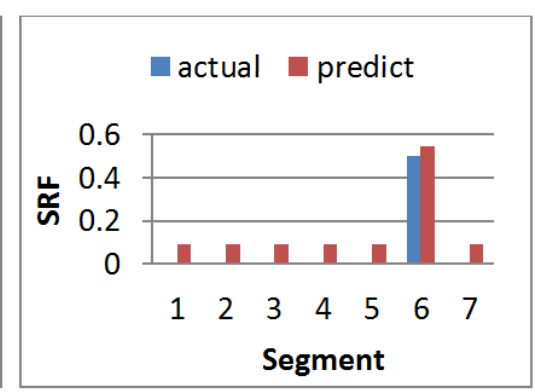

c) fgoalattain function

Figure 7. Performance of model updating for Case 2 using different function for default tolerance

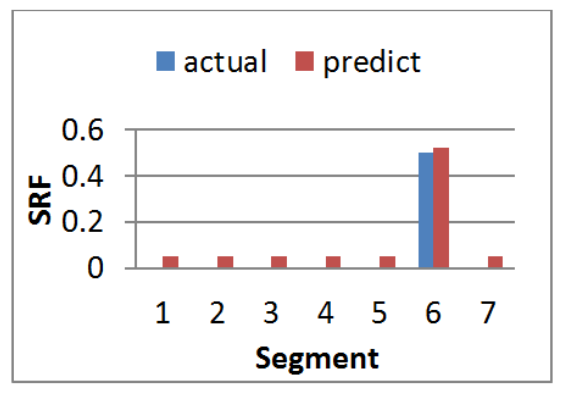

a) fmincon function

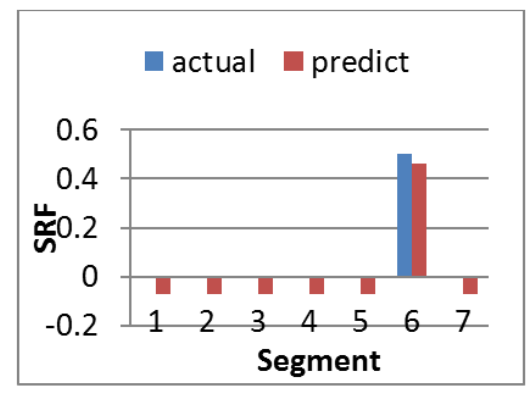

b) Isqnonlin function

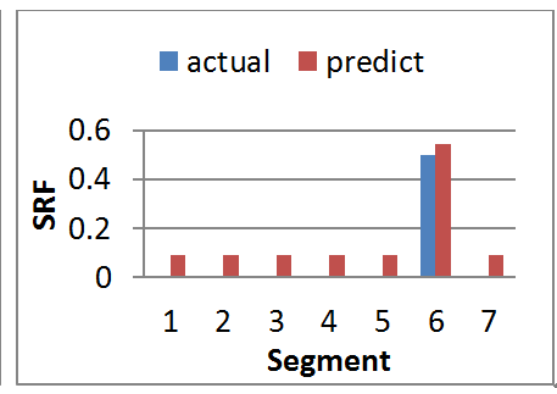

c) fgoalattain function

Figure 8. Performance of model updating for Case 2 using different function for higher tolerance

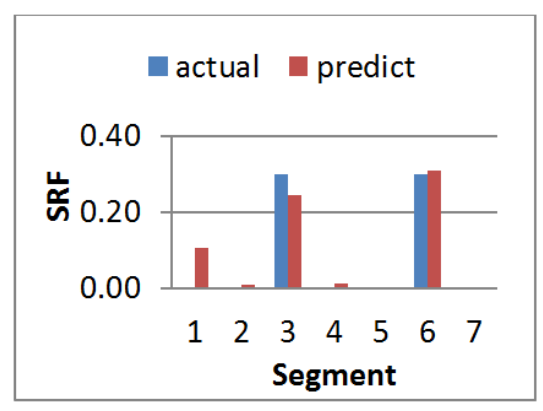

a) fmincon function

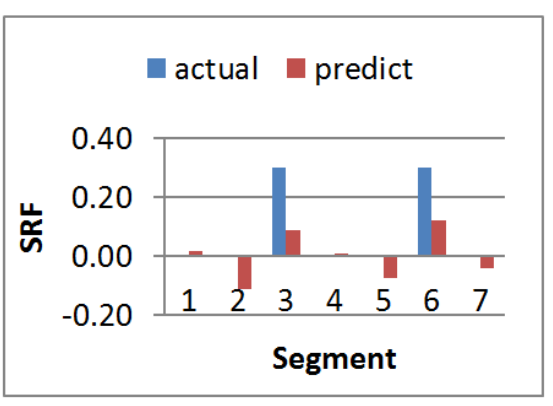

b) Isqnonlin function

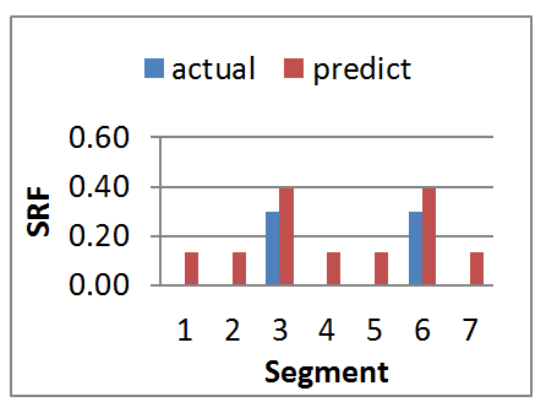

c) fgoalattain function

Figure 9. Performance of model updating for Case 3 using different function for default tolerance

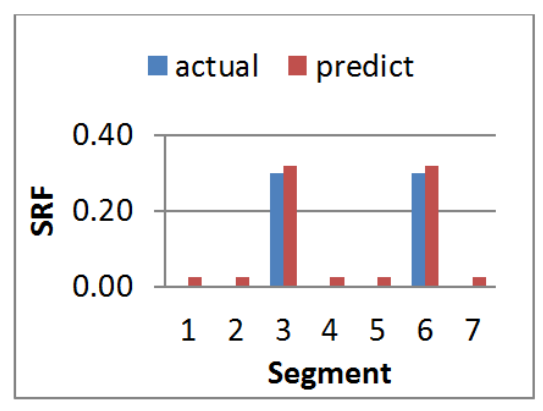

a) fmincon function

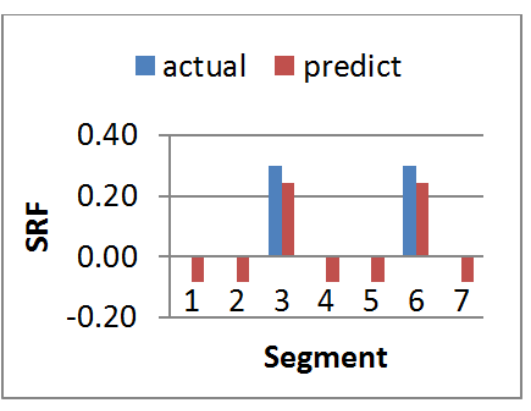

b) Isqnonlin function

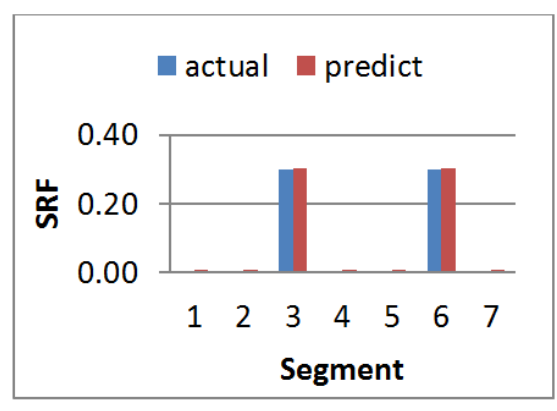

c) fgoalattain function

Figure 10. Performance of model updating for Case 3 using different function for higher tolerance 
The performance of model updating using different algorithms is evaluated based on the capability to predict the actual damage's location and damage severities. Based on Fig. 5(a), Fig. 7(a) and Fig. 9(a), it is observed that fmincon optimisation function can identify the damaged location correctly with small error of damage severities. However, false identification in other undamaged segments occurs in all cases with acceptable error. Using Isqnonlin optimisation function from Fig. 5(b), Fig. 7(b) and Fig. 9(b) show the location of damaged identified correctly but poorly in damaged severities. The highest error of $70 \%$ for Case 3 can be illustrated in Fig. 9(b). Nevertheless, the model is still capable to locate the damage due to the SRF of segments 3 and 6 is higher over other segments which is 0.09 and 0.12 , respectively.

Fig. 5(c), Fig. 7(c) and Fig. 9(c) demonstrate the ability of fgoalattain optimisation function to predict damaged location and severities. The locations are successfully identified with slightly overestimate the severities for all damaged cases. However, false identification for all undamaged segments is predicted with constant SRF value. According to [19], underestimates and overestimates of SRF value can be observed at undamaged segments due to numerical error and has been encountered by many researchers before. The trend for fmincon, Isqnonlin and fgoalattain performance is same for single damage cases (Case 1 and Case 2) and multiple damage case (Case 3 ).

Fig. 6, 8 and 10 depict the improvement of model updating performance after applying higher tolerance for different algorithms in all damaged cases. It can be seen that higher tolerance criteria provide more accurate result in locating damaged and predict damaged severities for all damage cases. The performance of different algorithms has obviously improved in locating the damaged location and predicted the damage severities for all damage cases as evidenced in Fig. 6, Fig. 8 and Fig 10. The false identification in undamaged segments with constant SRF value and smaller errors is observed for all cases and functions. Fig. 10(c) shows the result improves with great accuracy with small errors. According to [19], some minor overestimates and underestimates of SRF values at undamaged segments are due to numerical error and have been encountered by many researchers before.

However, this constant false identification has been noticed earlier using default tolerance in fgoalattain function. It indicates fgoalattain may provide more accurate result without applying higher tolerance. This finding is parallel to the study by [20] which uses fgoalattain function rather than others function for model updating process due to ability to give better result.

Fig. 10 presents the MSE for all three functions when the tolerance is increased for single damaged cases and multiple damaged cases, respectively. From Fig. 10(a), the MSE decreased with the increment of tolerance for cases employed fmincon and Isqnonlin functions. Thus, it indicates that the result improved when higher tolerance is applied for these fmincon and Isqnonlin function. However, in the damaged case that employed fgoalattain function, the MSE values recorded are observed to be inconsistent and did not show any improvement after 1e-8 tolerance is applied.

For multiple damaged cases, it is observed that MSE decreased as tolerance increased for all functions. The same trend is discovered for all three different functions in which increment of a number of iterations with higher tolerance is applied. The evidence is presented in Fig. 11. 


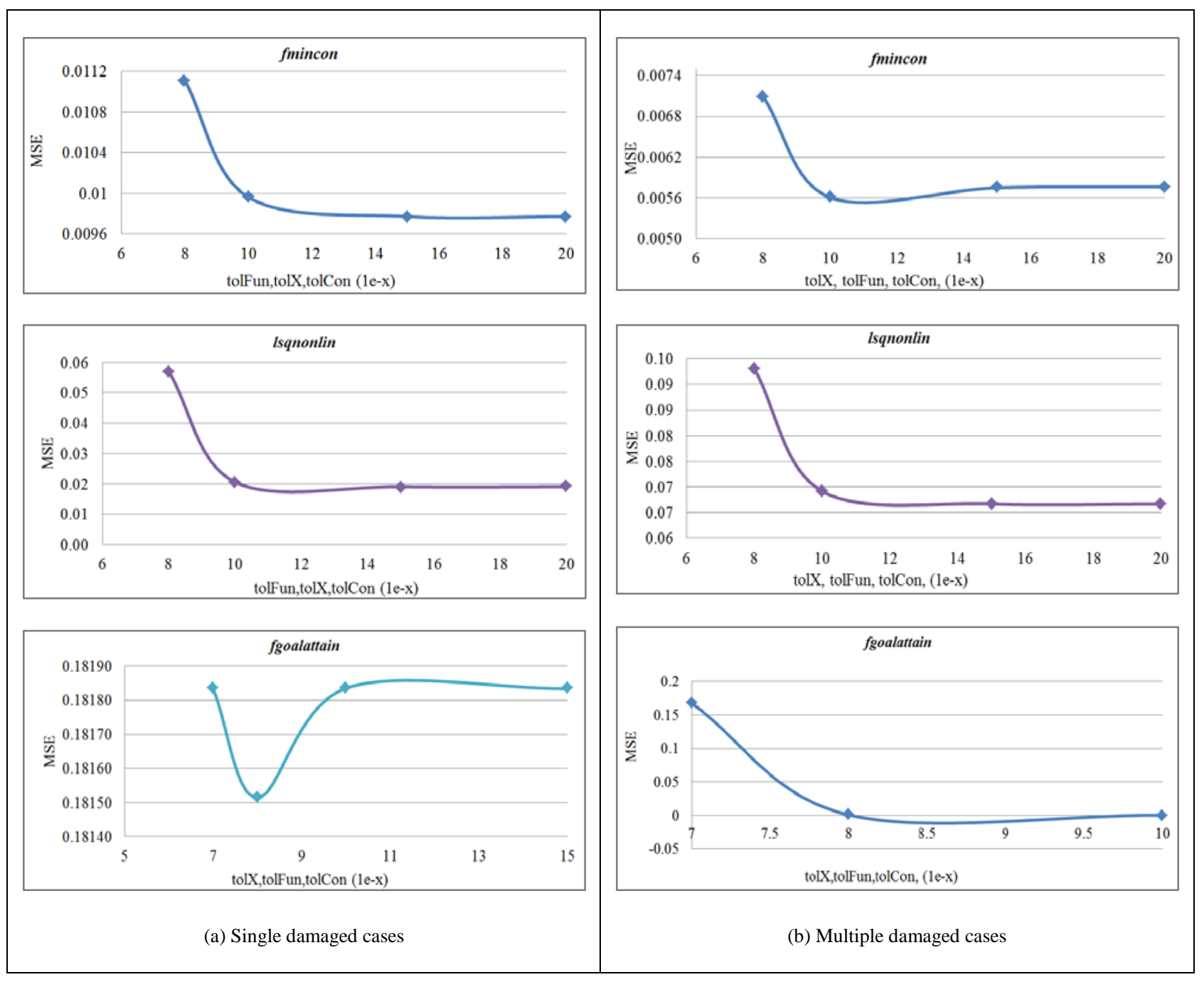

Figure 10. The MSE with different value of tolerance for three different functions 


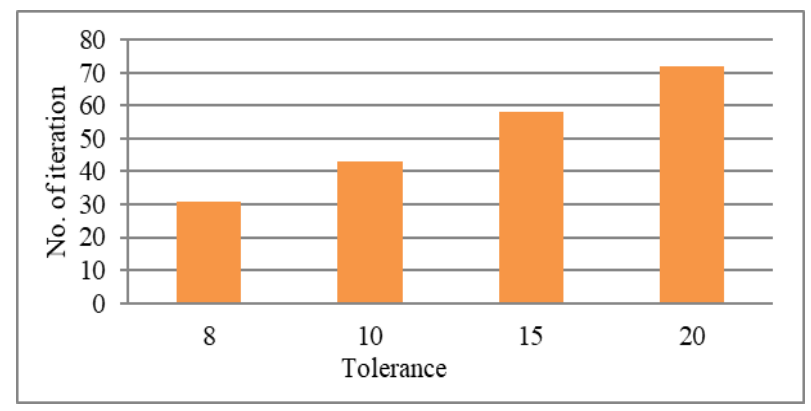

(a) fmincon

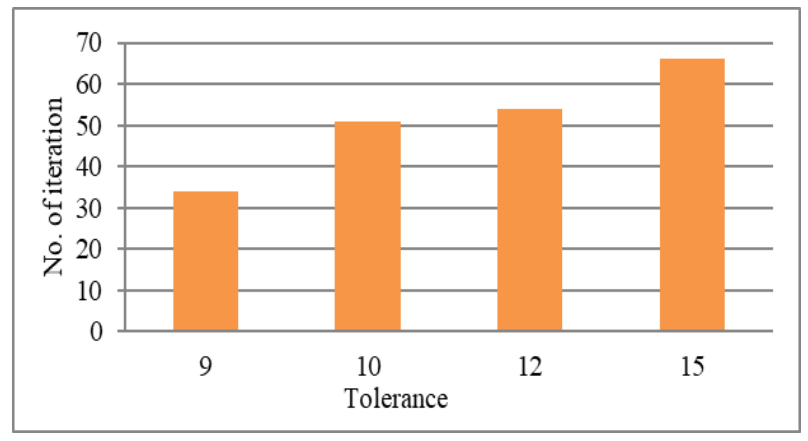

(b) Isqnonlin

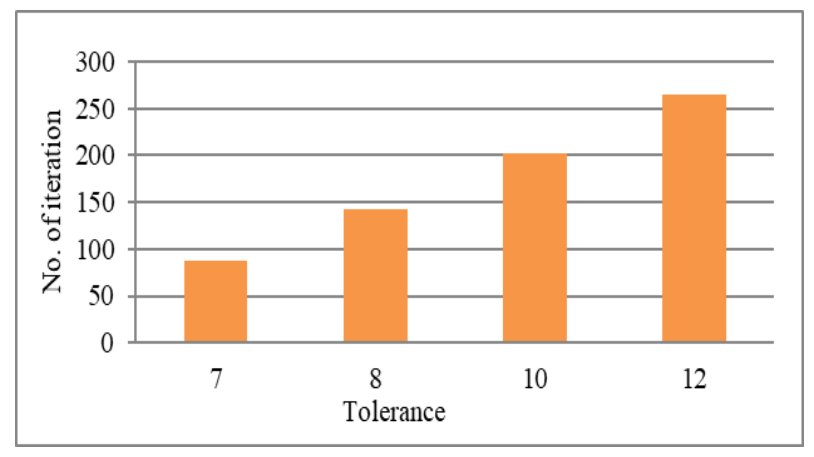

(c) fgoalattain

Figure 11. Number of iteration vs. Tolerance

\section{Conclusions}

This study demonstrates the comparative study of different algorithms using different optimisation functions which are constrained optimisation (fmincon), least-square optimisation (Isqnonlin) and multiobjective optimisation (fgoalattain) in damage detection. Three damage cases were investigated to verify the applicability of model updating to detect damaged in slab structure. Single damaged and multiple damaged were simulated using FEM and dynamic parameter of mode shapes obtained from finite element analysis was employed in this study. Higher tolerance was applied to all optimisation functions to demonstrate the effect of tolerance on the result performance. The conclusions that can be made based on the result are as follow:
1. Model updating using three optimisation functions of different algorithms used in this study was capable to provide reliable result in damage detection. However, the performance of fgoalattain optimisation function using default tolerance provided better result as compared to lsqnonlin optimisation function and fmincon optimisation function.

2. Dynamic properties of mode shapes generated from FEM utilised in the evaluation of MAC in the function of $E$ value may provide a good result in damage detection. However, study by [16] and [17] showed that the accuracy of the result can be improved by combination of natural frequencies and mode shapes in evaluation of the objective function.

3. It was observed that higher tolerance criteria can improve the performance of all the different optimisation functions in damage detection to detect damage location and predict the damage severities.

\section{Acknowledgments}

The authors would like to acknowledge the School of Civil Engineering, College of Engineering, Universiti Teknologi MARA, Cawangan Pulau Pinang, for providing the financial support for the publication of this research findings and the Faculty of Engineering, Universiti Teknologi Malaysia for providing the research materials for this research.

\section{REFERENCES}

[1] L. Andrade Nunes, R. Piazzaroli Finotti Amaral, F. D. Souza Barbosa and A. Abrahão Cury, A hybrid learning strategy for structural damage detection, Structural Health Monitoring, Vol.20, No.4, 2143-2160, 2021.

[2] C. R. Farrar and K. Worden, An introduction to structural health monitoring, Philosophical Transactions of the Royal Society A: Mathematical, Physical and Engineering Sciences, Vol.365, No.1851, 303-315, 2007.

[3] R. Hou and Y. Xia, Review on the new development of vibration-based damage identification for civil engineering structures: 2010-2019, Journal of Sound and Vibration, 115741, 2020.

[4] H. Nick, and A. Aziminejad, Vibration-based damage identification in steel girder bridges using Artificial Neural Network under noisy conditions. Journal of Nondestructive Evaluation, Vol.40, No.1, 1-22, 2021.

[5] J. E. Mottershead, M. Link, and M. I. Friswell, The sensitivity method in finite element model updating: A tutorial, Mechanical Systems and Signal Processing, Vol. 25, No.7, 2275-2296, 2011.

[6] C. P. Fritzen, D. Jennewein, and T. Kieffer, Damage detection based on model updating methods, Journal of 
Mechanical System and Signal Processing, Vol.12, No.1, 163:186, 1998.

[7] C. R. Farrar, S. W. Doebling and D. A. Nix, Vibration-based structural damage identification, Journal of Mathematical, Physical \& Engineering Sciences, Vol.359, No.1778, 131-149, 2001.

[8] F. Asma and A. Bouazzouni, Least squares finite element model updating, University Mouloud Mammeri, 2005.

[9] B. Hofmeister, M. Bruns and R. Rolfes, Finite element model updating using deterministic optimisation: A global pattern search approach, Engineering Structures, Vol.95, 373-381, 2019.

[10] P. E. Carden and P. Fanning, Vibration based condition monitoring: A review, Journal of Structural Health Monitoring, Vol.3, No.4, 355-357, 2004.

[11] S. W. Doebling, C. R. Farrar and M. B. Prime, A summary review of vibration-based damage identification methods, The Shock and Vibration Digest, Vol.30, No.2, 91-105, 1998.

[12] B. Caesar and J. Peter, Direct update of dynamic mathematical models from modal test data, AIAA Journal, Vol.25, No.11, 1987

[13] J. E. Mottershead, M. Link, and M. I. Friswell, Model updating in structural dynamics: A survey, Journal of Sound and Vibration, Vol.167, No.3, 347-375, 1993.
[14] J. E. Mottershead, M. Link, and M. I. Friswell, The sensitivity method in finite element model updating: A tutorial, Journal of Mechanical System and Signal Processing, Vol.25, No.7, 2275-2296, 2011.

[15] X. He, Vibration-based damage identification and health monitoring of civil structures, University of California, $\mathrm{PhD}$ Thesis, 2008.

[16] B. Jaishi and W. X. Ren, finite element model updating based on eigenvalue and strain energy residuals using multiobjective optimisation technique, Mechanical Systems and Signal Processing, Vol.21, No.5, 2295-2317, 2007.

[17] Y. X. Zhang, S.H. Sim and B.F. Spencer, finite element model updating of a truss using incomplete modal data, Shanghai Normal University and University of Illinois, Urbana, USA, 2008.

[18] Q. W. Zhang, C. C. Chang and T. Y. P. Chang, Finite element model updating for structures with parametric constraints, Earthquake Engineering \& Structural Dynamics, Vol.29, No.7, 927-944, 2000.

[19] N. Bakhary, structural condition monitoring and damage identification with Artificial Neural Network, University of Western Australia, PhD Thesis, 2008.

[20] S. Umar, Response Surface Methodology for damage detection using frequency and mode shapes, Universiti Teknologi Malaysia, Master Thesis, 2015. 